\title{
Étude de l'écoulement à travers une soupape de décharge de turbocompresseur
}

\author{
Mohamed Mseddi ${ }^{\mathrm{a}}$, Zied Driss, Mounir Baccar et Mohamed Salah Abid \\ Laboratoire des Systèmes Électro-Mécaniques (LASEM), Département de Génie Mécanique, École Nationale d'Ingénieurs \\ de Sfax (ENIS), Route de Soukra, BP <W>, 3038 Sfax, Tunisie
}

Reçu le 10 novembre 2003, accepté le 10 novembre 2004

\begin{abstract}
Résumé - Une étude de l'écoulement dans une soupape de décharge de turbocompresseur de suralimentation, est présentée dans ce travail. Une modélisation numérique tridimensionnelle de l'écoulement turbulent à travers la soupape a été entreprise en s'appuyant sur la résolution des équations de Navier-Stokes et d'énergie couplées à un modèle de turbulence de type $k-\varepsilon$. Cette modélisation a permis de fournir une connaissance fine des structures de l'écoulement à travers la soupape, et de prédire ses courbes caractéristiques de fonctionnement. À partir des résultats locaux de l'écoulement, le coefficient de débit de la soupape a été déterminé en fonction de sa levée. De même, à partir des données géométriques de la soupape, une modélisation analytique a été établie, en vue de donner le débit en fonction du taux de détente. Pour valider aussi bien le calcul par voie de simulation numérique que celui fourni par l'approche analytique proposée, nous avons utilisé un banc d'essai équipé d'un turbocompresseur à soupape de décharge pour automobile. La comparaison des courbes caractéristiques de fonctionnement obtenues à partir de notre travail avec celles des travaux expérimentaux antérieurs, a montré une bonne concordance.
\end{abstract}

Mots clés : Soupape / turbocompresseur / fluide compressible / écoulement turbulent / modélisation numérique / modèle $k-\varepsilon$ / modélisation analytique / courbe caractéristique de fonctionnement / coefficient de débit

\begin{abstract}
Study of flow through a turbocharger wastegate. A study of flow through a turbocharger wastegate for automotive engines is presented. A three-dimensional analysis of the aerodynamic and thermal characteristics of the turbulent flow over the wastegate is developed using Navier-Stokes and energy equations in conjunction with the standard $k-\varepsilon$ turbulence model. The numerical model permits prediction of the flow and turbulence characteristics, the working curves and the discharge coefficient of the wastegate. An analytic model resulting in equation giving the mass rate of the wastegate is also presented by using its geometry characteristics. Finally, the numerical and analytic results are compared to our experimental results and those obtained by other authors. The comparison proved a good agreement.
\end{abstract}

Key words: Wastegate / turbocharger / compressible fluid / turbulent flow / numerical model / $k-\varepsilon$ model / analytic model / working curves / discharge coefficient

\section{Introduction}

Lors du développement d'un projet de moteur à combustion interne suralimenté par turbocompresseur, la connaissance des performances de la turbine de suralimentation est nécessaire. D'autre part, aux forts régimes moteurs, et dans le cas d'une turbine munie d'une soupape de décharge pour la régulation de la pression de suralimentation, les courbes caractéristiques de fonctionnement de la soupape sont indispensables.

\footnotetext{
a Auteur correspondant : Mohamed.Mseddi@fss.rnu.tn
}

La soupape de décharge appelée encore «wastegate » fait corps avec la turbine de suralimentation. Elle permet de limiter la pression de suralimentation à une valeur maximale prédéfinie et d'éviter ainsi la détérioration du moteur. En effet, au-delà d'un certain régime du moteur suralimenté, la pression à la sortie du compresseur dépasse une certaine valeur préréglée et admissible par le moteur. À cet effet, la soupape de décharge s'ouvre, et une partie des gaz d'échappement est dérivée directement vers la tubulure d'échappement.

Un grand nombre de travaux de recherches sur les turbines de suralimentation à clapet ou à soupape 


\section{Nomenclature}

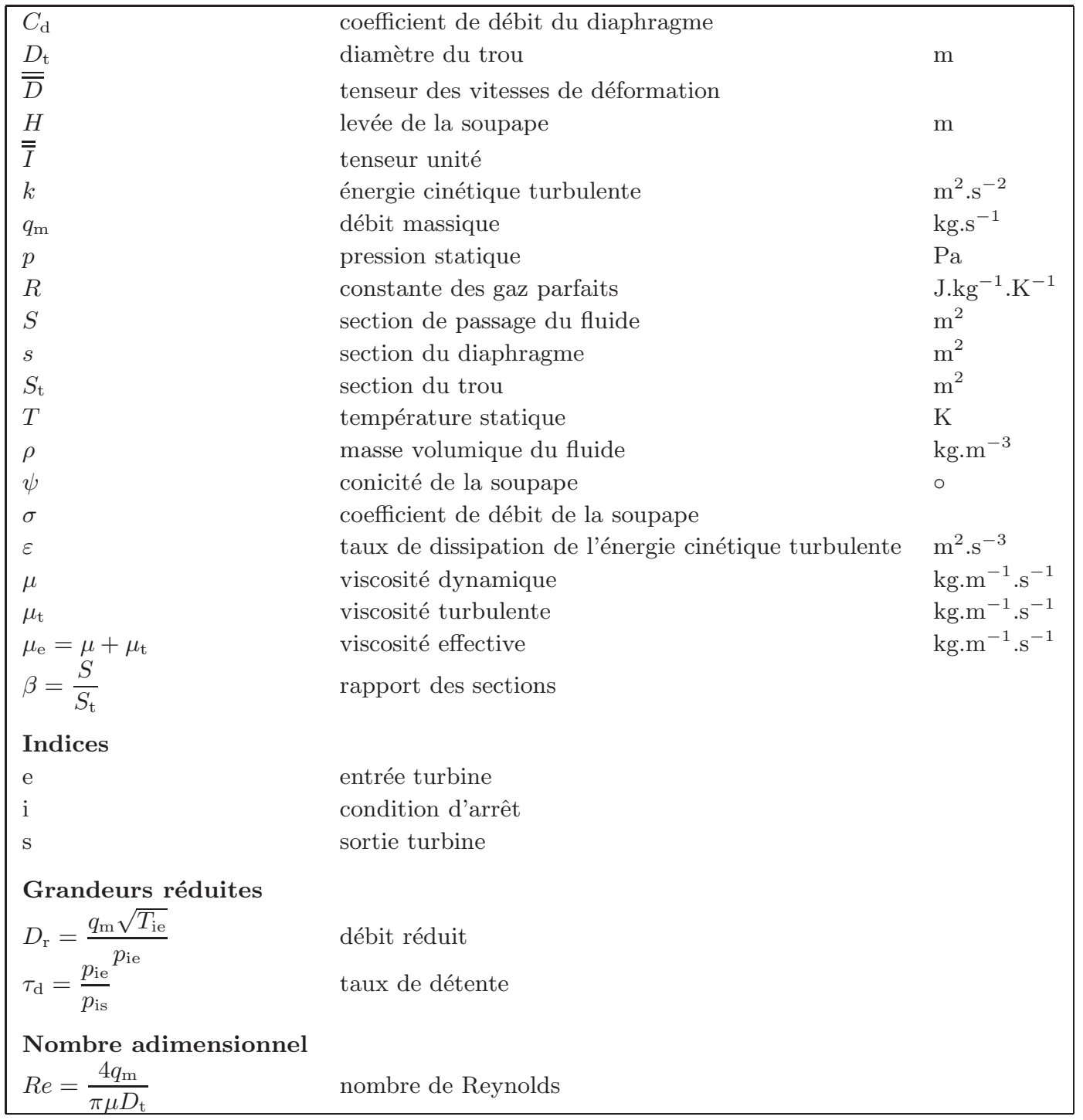

de décharge, ont été publiés. On peut citer ceux de Boussarhane [1], Schorn et al. [2], Jullien et al. [3], Thomas [4], Friberg et al. [5], Fèvre [6], Lavy et al. [7], Mseddi [8], Frelin [9], Frelin et Podevin [10], Hammoud [11], Podevin et al. [12,13] et Mseddi et al. [14].

Cependant, dans la plupart des travaux antérieurs se rapportant à l'étude des turbines de suralimentation, la soupape est supposée fermée.

Dans ce travail, nous présentons une étude de l'écoulement turbulent d'un gaz parfait à travers une soupape de décharge de type NW20 équipant un turbocompresseur de suralimentation de la firme Kühnle, Kopp et Kausch (KKK) (Fig. 1), qui a d'ailleurs fait l'objet de plusieurs travaux de recherches théoriques et expérimentaux antérieurs (Friberg et al. [5], Mseddi [8], Frelin [9] et Bardez [15]). Dans son travail se rapportant à l'étude du turbocompresseur KKK, Bardez [15] a déterminé les courbes caractéristiques de fonctionnement de la soupape de décharge, par voie expérimentale.
Nous proposons alors une étude de l'écoulement à travers cette soupape par différentes approches numérique, analytique et expérimentale. Nous supposerons que la soupape fonctionne dans les mêmes conditions de pression que la turbine.

La modélisation numérique de l'écoulement turbulent à travers la soupape de décharge rendra possible la compréhension de son fonctionnement et permettra de :

- fournir une connaissance finalisée des comportements aérodynamique et thermique de l'écoulement à travers la soupape, à savoir les champs de vitesse, de pression et de température, ainsi que les caractéristiques turbulentes,

- prédire à partir de cette étude, la courbe caractéristique de fonctionnement pour une levée quelconque de la soupape,

- déterminer l'évolution du coefficient de la soupape en fonction de la levée. 


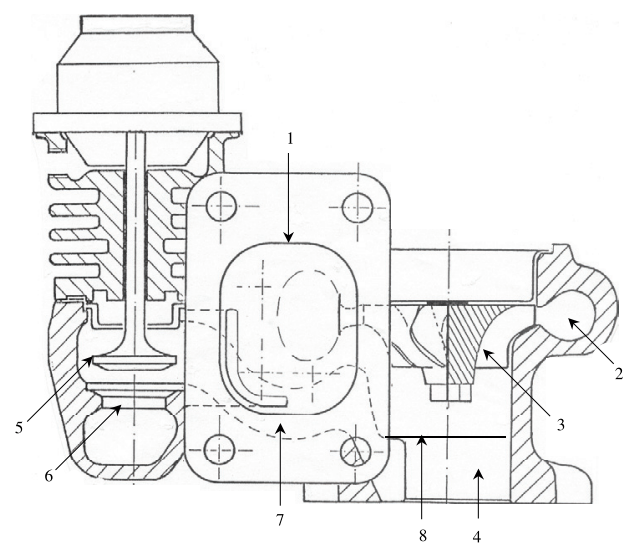

\begin{tabular}{|c|l|}
\hline $\mathbf{1}$ & Entrée turbine \\
\hline $\mathbf{2}$ & Volute \\
\hline $\mathbf{3}$ & Roue mobile \\
\hline $\mathbf{4}$ & Diffuseur \\
\hline $\mathbf{5}$ & Soupape \\
\hline $\mathbf{6}$ & Orifice de la soupape \\
\hline $\mathbf{7}$ & Canal des gaz by-passés \\
\hline $\mathbf{8}$ & Obturateur \\
\hline
\end{tabular}

Fig. 1. Vue de la turbine KKK K14 à soupape de décharge NW20.

Cette modélisation s'appuie sur la résolution des équations de Navier-Stokes et d'énergie couplées à un modèle de turbulence de type $k-\varepsilon$ standard. La résolution est effectuée par le code commercial Fluent (version 5.6).

D'autre part, une approche analytique ayant pour objectif d'établir une équation simple qui traduit les caractéristiques de fonctionnement de la soupape, sera présentée. Cette équation permettra, à partir des données géométriques de la soupape, de calculer son débit pour un taux de détente et une levée donnés.

Enfin, nous validons les résultats théoriques obtenus moyennant un banc d'essai équipé par un turbocompresseur de type KKK à soupape de décharge de type NW20.

\section{Modélisation numérique}

\subsection{Géométrie et conditions aux limites}

La géométrie tridimensionnelle de la conduite de dérivation des gaz et de la soupape ainsi que le maillage tridimensionnel du domaine de calcul sont développés moyennant un logiciel commercial « Gambit ». Le maillage utilisé comporte 46273 cellules tétraédriques.

D'autre part, pour les conditions aux limites, nous imposons le débit massique et la température totale des gaz à l'entrée, ainsi que la pression statique à la sortie.

Pour les conditions de turbulence, nous spécifions l'intensité de la turbulence $(4 \%)$ et le diamètre hydraulique (diamètre du trou $D_{\mathrm{t}}=19 \mathrm{~mm}$ ). L'air étant supposé parfait, ses propriétés sont obtenues à partir de la théorie cinétique des gaz.

\section{2 Équations de Navier-Stokes moyennes}

Les équations de Navier-Stokes moyennes décrivent la composante moyenne statistique des écoulements turbulents ; le champ turbulent instantané étant classiquement décomposé en une composante moyenne et une composante fluctuante de moyenne nulle.
Pour un fluide compressible, ces équations se présentent sous la forme conservative suivante :

$$
\begin{gathered}
\frac{\partial \rho}{\partial t}+\operatorname{div}(\rho \vec{V})=0 \\
\frac{\partial(\rho \vec{V})}{\partial t}+\operatorname{div}(\rho \vec{V} \otimes \vec{V})=\operatorname{div}\left(p \overline{\bar{I}}+\overline{\bar{\tau}}+\overline{\bar{\tau}}_{\mathrm{R}}\right) \\
\frac{\partial(\rho E)}{\partial t}+\operatorname{div}(\rho E \vec{V})=\operatorname{div}\left[\left(p \overline{\bar{I}}+\overline{\bar{\tau}}+\overline{\bar{\tau}}_{\mathrm{R}}\right) \cdot \vec{V}\right]
\end{gathered}
$$

Dans ces expressions, $\rho, \vec{V}$ et $p$ désignent les valeurs moyennes de la masse volumique, du vecteur vitesse et de la pression statique. Pour $\rho$ et $p$, il s'agit d'une valeur moyenne statistique; en revanche la valeur moyenne de la vitesse est définie avec une pondération par la masse volumique. Cette approximation est également appliquée pour la définition de l'énergie interne spécifique $e$. L'énergie moyenne totale $E$ est alors la somme de l'énergie totale du mouvement moyen : $e+\frac{1}{2} V^{2}$ et de l'énergie cinétique du mouvement turbulent $k$.

Pour un gaz parfait à chaleurs spécifiques constantes de rapport $\gamma=\frac{C_{p}}{C_{v}}$, la pression statique est donnée par la loi d'état des gaz parfaits :

$$
p=\rho R T=(\gamma-1) \rho e
$$

Le tenseur $\overline{\bar{\tau}}$ représente la valeur moyenne du tenseur des contraintes.

Par rapport aux équations de Navier-Stokes instantanées, les équations moyennes font apparaître deux termes supplémentaires : l'énergie cinétique turbulente $k$ intervenant dans l'expression de l'énergie totale $E$, et le tenseur des contraintes de Reynolds $\overline{\bar{\tau}}_{\mathrm{R}}$. L'expression de ces deux quantités en fonction du champ moyen est donnée par le modèle de turbulence de type $k-\varepsilon$ développé ci-après. 
Tableau 1. Constantes du modèle $(k, \varepsilon)$ standard.

\begin{tabular}{ccccc}
\hline$C_{1}$ & $C_{2}$ & $C_{\mu}$ & $\sigma_{\mathrm{k}}$ & $\sigma_{\varepsilon}$ \\
\hline 1,44 & 1,92 & 0,09 & 1 & 1,3 \\
\hline
\end{tabular}

\subsection{Modèle de turbulence}

Le tenseur des contraintes de Reynolds $\overline{\bar{\tau}}_{\mathrm{R}}$ est relié aux gradients de vitesses moyennes par :

$$
\overline{\bar{\tau}}_{\mathrm{R}}=-\frac{2}{3}\left(\rho k+\mu_{\mathrm{t}} \operatorname{div} \vec{V}\right) \overline{\bar{I}}+2 \mu_{\mathrm{t}} \overline{\bar{D}}
$$

La viscosité turbulente $\mu_{\mathrm{t}}$ est donnée par :

$$
\mu_{\mathrm{t}}=C_{\mu} \rho \frac{k^{2}}{\varepsilon}
$$

Cette viscosité est fonction de l'énergie cinétique turbulente $k$ et de son taux de dissipation $\varepsilon$.

Le modèle de turbulence choisi est du type $k-\varepsilon$ standard (Launder et Spalding [16]). Les équations d'énergie cinétique turbulente $k$ et de son taux de dissipation $\varepsilon$ s'écrivent :

$$
\frac{\partial(\rho k)}{\partial t}+\operatorname{div}(\rho \vec{V} k)=\operatorname{div}\left[\left(\mu+\frac{\mu_{\mathrm{t}}}{\sigma_{\mathrm{k}}}\right) \nabla k\right]+\overline{\bar{\tau}}_{\mathrm{R}}: \nabla \vec{V}-\rho \varepsilon
$$

$$
\begin{aligned}
& \frac{\partial(\rho \varepsilon)}{\partial t}+\operatorname{div}(\rho \vec{V} \varepsilon)= \\
& \quad \operatorname{div}\left[\left(\mu+\frac{\mu_{\mathrm{t}}}{\sigma_{\varepsilon}}\right) \nabla \varepsilon\right]+C_{1} \frac{\varepsilon}{k} \overline{\bar{\tau}}_{\mathrm{R}}: \nabla \vec{V}-C_{2} \rho \frac{\varepsilon^{2}}{k}
\end{aligned}
$$

avec $C_{1}, C_{2}, C_{\mu}, \sigma_{\mathrm{k}}$ et $\sigma_{\varepsilon}$, les constantes du modèle $(k$, $\varepsilon)$ standard. Ces constantes sont répertoriées dans le tableau 1.

\subsection{Traitement à proximité des parois}

Pour prédire la contrainte à la paroi, nous avons utilisé une loi logarithmique universelle (Launder et Spalding [17]), qui suppose que le premier nœud du maillage, avoisinant les parois solides, est à une distance $y$ suffisante pour que l'on se trouve dans la sous-couche turbulente.

Dans ces conditions, la loi de paroi s'écrit :

$$
\frac{V}{V_{0}}=\frac{1}{\kappa} \log \left(E \frac{\rho y V_{0}}{\mu}\right)
$$

où : $\kappa$, la constante de Von Karman $(=0,42), E$, une constante empirique $(=9,81), V_{0}$ et $V$ respectivement, la vitesse de frottement et la vitesse adjacente à la paroi.

L'expression (9) est valable pour : $\frac{\rho y V_{0}}{\mu}>11,225$.

La vitesse de frottement $V_{0}$ est déterminée par voie itérative et la contrainte à la paroi $\tau_{\mathrm{p}}$ s'obtient :

$$
\tau_{\mathrm{p}}=\rho V_{0}^{2}
$$

\subsection{Résultats}

Nous allons étudier les structures de l'écoulement à travers la soupape de décharge objet de cette étude. Nous déterminons les distributions de vitesse, de pression et de température, ainsi que les caractéristiques locales de la turbulence, telles que l'énergie cinétique turbulente, son taux de dissipation ainsi que la viscosité turbulente.

Les résultats sont présentés dans deux plans différents : l'un contenant le canal d'amenée des gaz vers la soupape, et l'autre contenant le canal des gaz by-passés.

Les caractéristiques locales de l'écoulement ainsi que celles de la turbulence sont fournies pour une levée de $8 \mathrm{~mm}$ (ouverture maximale de la soupape), un débit $q_{\mathrm{m}}$ de 50 g.s ${ }^{-1}$, un taux de détente $\tau_{\text {d }}$ de 1,54 , et un nombre de Reynolds Re de $1,87 \times 10^{5}$.

Pour montrer l'influence de la levée et du débit sur ces caractéristiques, nous présentons dans le plan contenant le canal des gaz by-passés, des résultats pour une levée de $1,6 \mathrm{~mm}$ (20\% de l'ouverture maximale de la soupape), un débit $q_{\mathrm{m}}$ de 30 g.s ${ }^{-1}$ et un nombre de Reynolds Re de $1,12 \times 10^{5}$.

\subsubsection{Caractéristiques locales de l'écoulement}

Les figures $2 \mathrm{a}$ et $2 \mathrm{~b}$ présentent la répartition de la résultante des vitesses moyennes respectivement dans le plan contenant le canal des gaz by-passés, et dans le plan contenant le canal d'amenée des gaz vers la soupape, pour une levée de $8 \mathrm{~mm}$ et un débit de $50 \mathrm{~g} . \mathrm{s}^{-1}$. D'après ces figures, l'écoulement change de vitesse et de direction avant de contourner la soupape. D'autre part, l'écoulement d'air s'accélère à l'engagement dans l'entrefer soupape-carter à cause de la diminution de la section de l'écoulement.

Vers la sortie du canal des gaz by-passés, le fluide s'accélère de nouveau et forme un jet puissant atteignant une vitesse maximale de l'ordre de $160 \mathrm{~m} . \mathrm{s}^{-1}$.

Pour la même levée ( $8 \mathrm{~mm})$, la figure $2 \mathrm{c}$ montre l'influence de la diminution du débit sur la vitesse. On remarque que l'intensité de la vitesse diminue alors que la répartition ne varie que légèrement.

L'influence de la diminution de la levée sur la vitesse pour un même débit de 50 g.s ${ }^{-1}$, est montrée sur la figure $2 \mathrm{~d}$. D'après cette figure, la répartition de la vitesse change par rapport à celle présentée sur la figure $2 \mathrm{a}$. C'est dans l'entrefer soupape-carter que le fluide atteigne sa vitesse maximale $\left(\sim 200 \mathrm{~m} \cdot \mathrm{s}^{-1}\right)$. Vers la sortie du canal des gaz by-passés, le fluide s'accélère et forme également un jet dont l'intensité est inférieure à la valeur maximale.

D'autre part, la répartition de la pression statique est indiquée sur les cartographies de la figure 3. Dans le canal d'amenée des gaz vers la soupape, la pression atteint ses valeurs les plus élevées $\left(\sim 1,47 \times 10^{5} \mathrm{~Pa}\right)$, puis diminue en s'approchant de la soupape (Fig. 3b). Au niveau de la soupape, c'est la face de la tête de la soupape qui est soumise à la pression la plus élevée $\left(\sim 1,42 \times 10^{5} \mathrm{~Pa}\right)$. En passant dans l'entrefer, la pression diminue. Dans le reste de la conduite de dérivation, la pression décroît et atteint sa valeur minimale vers la sortie. 

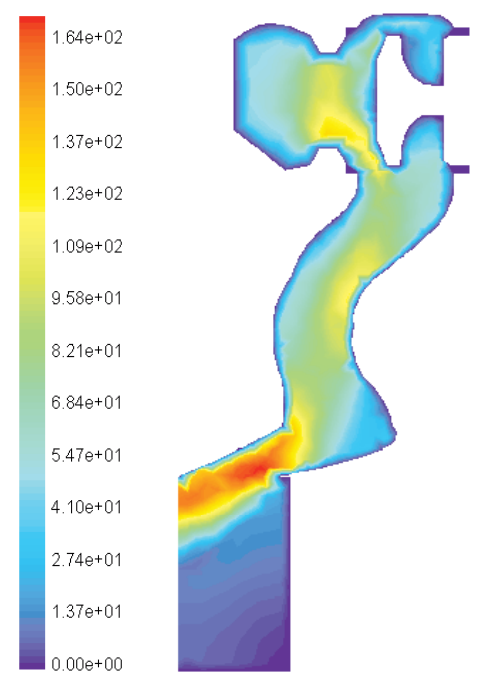

(a) Levée $8 \mathrm{~mm}$ - Débit $50 \mathrm{~g} / \mathrm{s}$
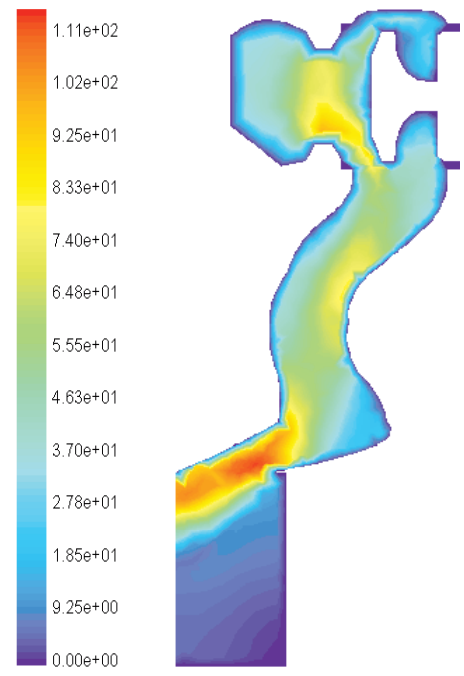

(c) Levée $8 \mathrm{~mm}$ - Débit $30 \mathrm{~g} / \mathrm{s}$
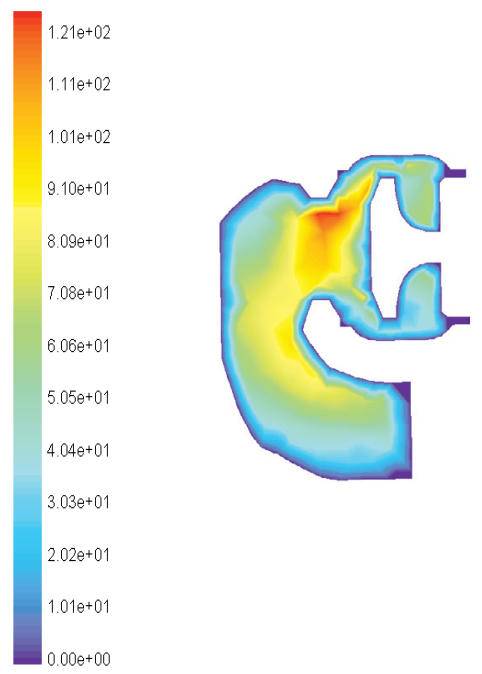

(b) Levée $8 \mathrm{~mm}$ - Débit $50 \mathrm{~g} / \mathrm{s}$

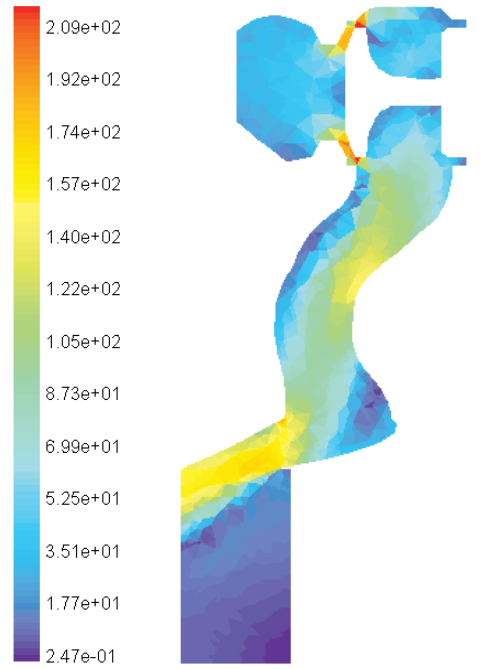

(d) Levée 1,6 mm - Débit $50 \mathrm{~g} / \mathrm{s}$

Fig. 2. Isocontours de la résultante des vitesses $\left(\mathrm{m} \cdot \mathrm{s}^{-1}\right)$.

Quant à l'influence du débit sur la pression, à levée constante, la figure $3 \mathrm{c}$ montre une répartition similaire à celle présentée sur la figure 3a, mais les valeurs de la pression sont moins élevées (valeur maximale de 1,19× $\left.10^{5} \mathrm{~Pa}\right)$.

D'autre part, d'après la figure $3 \mathrm{~d}$, une diminution de la levée, à débit constant, entraîne une augmentation importante de la pression statique, aussi bien dans le canal d'amenée des gaz vers la soupape, que dans le canal des gaz by-passés. Aussi, on assiste à une diminution brusque et rapide de la pression en passant dans l'entrefer soupape-carter, et un fort gradient de pression s'établit entre l'amont et l'aval de la soupape.

Pour ce qui concerne la température statique, la figure 4 montre qu'en s'approchant de la soupape, la température diminue. D'autre part, sur la tête de la soupape, la répartition de la température n'est pas uniforme. La température augmente ensuite dans le canal des gaz by-passés, puis diminue vers la sortie.

Pour une levée de $8 \mathrm{~mm}$ et un débit de 30 g.s ${ }^{-1}$, la figure 4c montre que la répartition de la température n'a presque pas varié par rapport à celle qui correspond à une levée de $8 \mathrm{~mm}$ et un débit de 50 g.s ${ }^{-1}$.

De même, pour un débit de 50 g.s ${ }^{-1}$ et une levée de 1,6 mm (Fig. 4d), la face de la tête de la soupape est soumise à une température plus élevée $(\sim 285 \mathrm{~K})$ et plus uniforme que celle correspondant à une levée de $8 \mathrm{~mm}$ (Fig. 4a). La valeur la plus faible de température ( $240 \mathrm{~K})$ est située vers la sortie du canal des gaz bypassés.

En conclusion, d'après les figures 2, 3 et 4, les répartitions de la résultante des vitesses, de la pression 

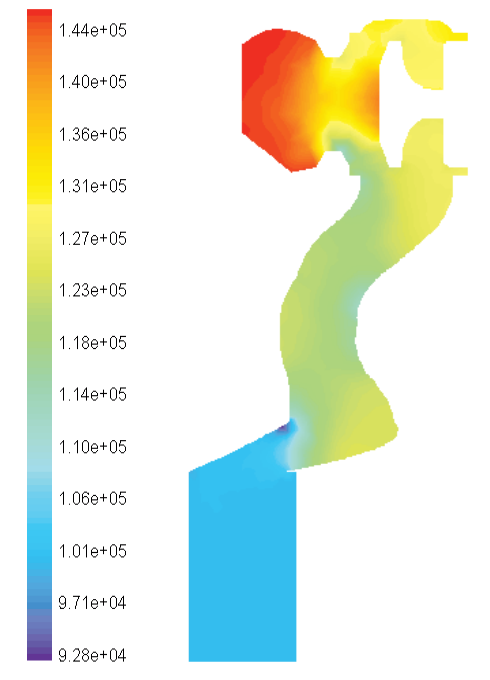

(a) Levée $8 \mathrm{~mm}$ - Débit $50 \mathrm{~g} / \mathrm{s}$
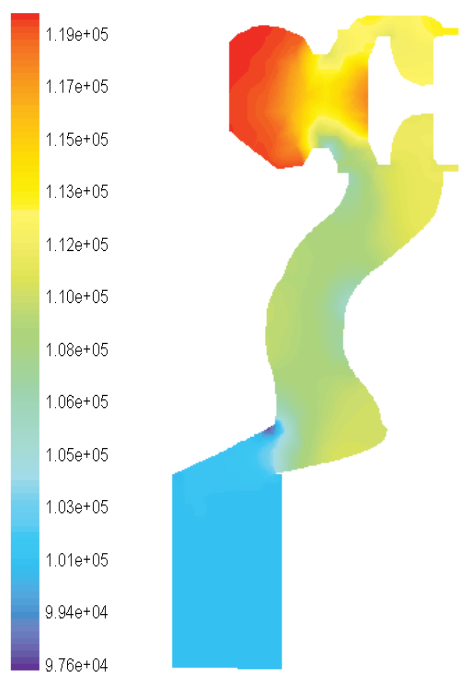

(c) Levée $8 \mathrm{~mm}$ - Débit $30 \mathrm{~g} / \mathrm{s}$
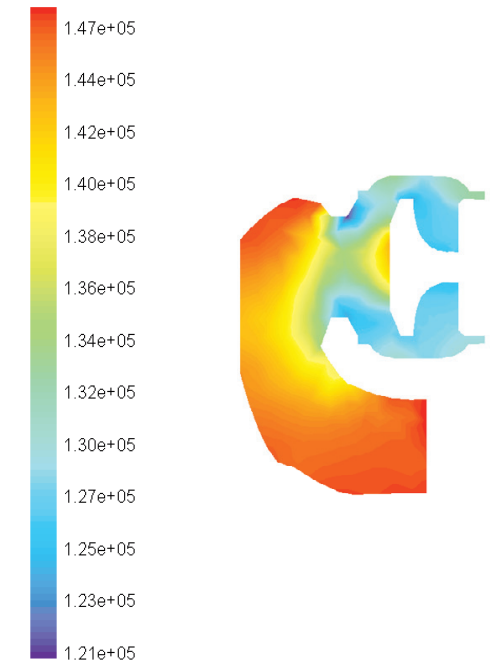

(b) Levée $8 \mathrm{~mm}$ - Débit $50 \mathrm{~g} / \mathrm{s}$

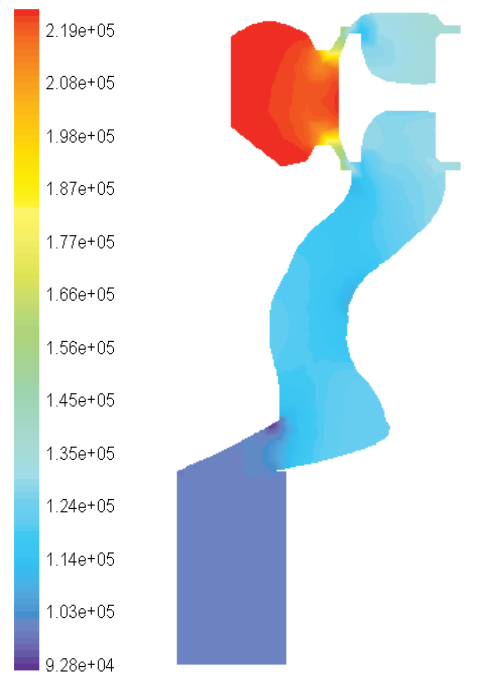

(d) Levée 1,6 mm - Débit $50 \mathrm{~g} / \mathrm{s}$

Fig. 3. Isocontours de la pression statique $(\mathrm{Pa})$.

statique et de la température statique dépendent fortement du taux d'ouverture de la soupape.

\subsubsection{Caractéristiques de la turbulence}

La connaissance détaillée de la structure fine de la turbulence permet de situer les régions soumises à des fluctuations importantes de vitesse.

Sur les figures $5 \mathrm{a}$ et $5 \mathrm{~b}$, la répartition de l'énergie cinétique turbulente $k$ est présentée pour une levée de $8 \mathrm{~mm}$ et un débit de 50 g.s ${ }^{-1}$. Il apparaît que l'énergie cinétique turbulente est très élevée sur la face de la tête de la soupape ainsi que dans l'entrefer, puis décroît en s'approchant de la sortie. Aussi bien dans le canal des gaz bypassés que dans le canal d'amenée des gaz vers la soupape, les valeurs de $k$ sont faibles. Ce même comportement est observé sur la figure 5c pour un débit plus faible.

En revanche, on assiste à un grand changement de la répartition de $k$ suite à une diminution de l'ouverture de la soupape. En effet, pour une levée de 1,6 mm et un débit de 50 g.s ${ }^{-1}$ (Fig. 5d), la région siège des valeurs élevées de $k\left(k_{\max } \sim 9,64 \times 10^{2} \mathrm{~m}^{2} . \mathrm{s}^{-2}\right)$ est située à la sortie de l'entrefer.

On note également qu'en diminuant le débit de 50 g.s à $^{-1} 0$ g.s. ${ }^{-1}$, la valeur maximale de l'énergie cinétique turbulente a presque diminué de moitié $\left(k_{\max }\right.$ passe de $8,47 \times 10^{2}$ à $\left.4,53 \times 10^{2} \mathrm{~m}^{2} \cdot \mathrm{s}^{-2}\right)$.

La connaissance de la distribution du taux de dissipation de l'énergie cinétique turbulente $\varepsilon$ permet de localiser les régions où la dissipation de l'énergie cinétique turbulente est maximale, c'est-à-dire celles où les fluctuations de vitesses sont les plus importantes. 


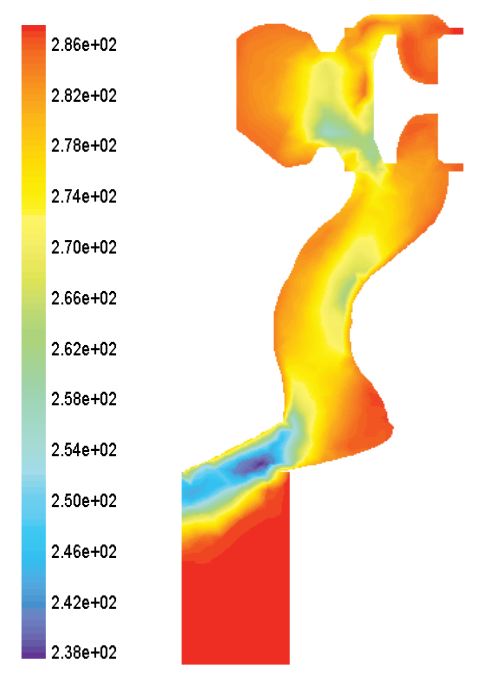

(a) Levée $8 \mathrm{~mm}$ - Débit $50 \mathrm{~g} / \mathrm{s}$

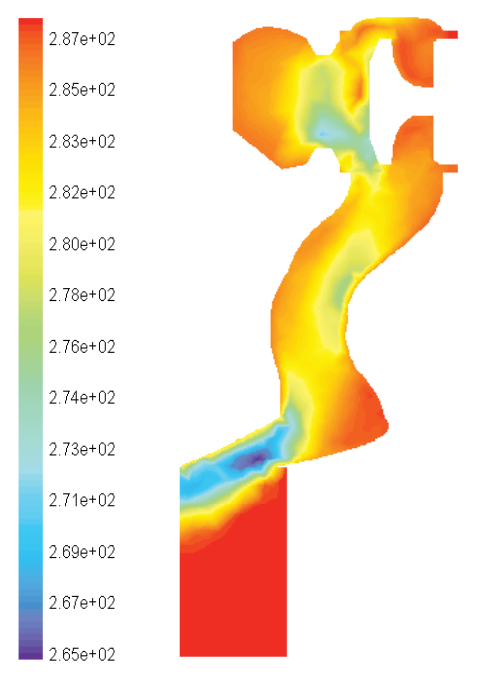

(c) Levée $8 \mathrm{~mm}$ - Débit $30 \mathrm{~g} / \mathrm{s}$
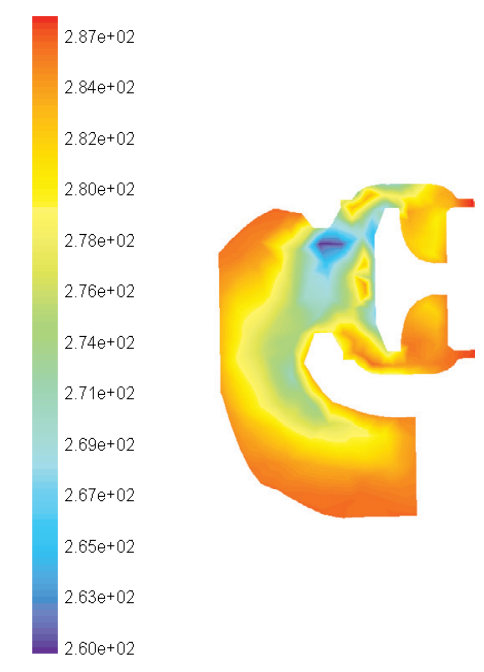

(b) Levée $8 \mathrm{~mm}$ - Débit $50 \mathrm{~g} / \mathrm{s}$

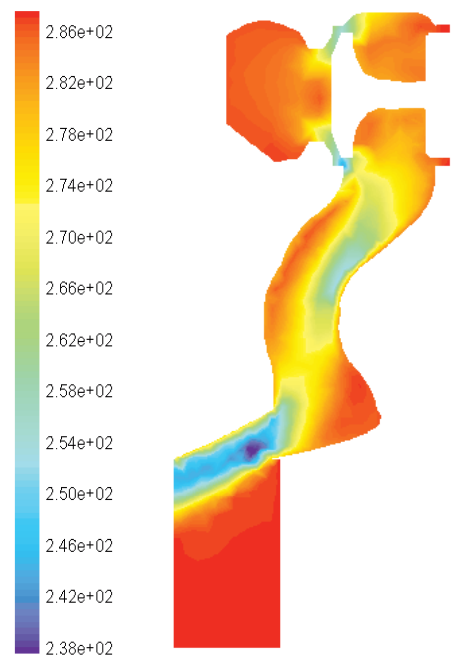

(d) Levée 1,6 mm - Débit $50 \mathrm{~g} / \mathrm{s}$

Fig. 4. Isocontours de la température statique $(\mathrm{K})$.

La figure 6 montre que les zones de fortes énergies cinétiques turbulentes sont également le siège de fortes dissipations. En particulier, il apparaît que les zones sièges de forts taux de dissipation de l'énergie cinétique turbulente $\varepsilon$ prennent naissance dans la zone proche de la tête de la soupape, et s'étendent un peu vers l'aval. Ce comportement montre clairement l'intérêt pratique d'utiliser différentes géométries de soupape dans un but de réduire la dissipation au niveau de leur couronne.

Pour une levée de $8 \mathrm{~mm}$, et pour deux valeurs différentes du débit : $50 \mathrm{~g} . \mathrm{s}^{-1}$ et $30 \mathrm{~g} . \mathrm{s}^{-1}$, la valeur de $\varepsilon$ est maximale sur la face de la tête de la soupape, et vaut respectivement $3,28 \times 10^{7}$ et $1,21 \times 10^{7} \mathrm{~m}^{2} . \mathrm{s}^{-3}$. Cependant, pour une levée de $1,6 \mathrm{~mm}$, la valeur de $\varepsilon$ est maximale dans l'entrefer de la soupape.

Dans le reste de la conduite de dérivation, $\varepsilon$ atteint rapidement des valeurs très faibles.
La figure 7 permet de visualiser la répartition de la viscosité effective. Il apparaît que cette viscosité atteint des valeurs importantes $\left(\sim 1,40 \times 10^{-2} \mathrm{~kg} \cdot \mathrm{m}^{-1} \cdot \mathrm{s}^{-1}\right)$ en amont de la tête de la soupape, ainsi que dans le courant de décharge.

Tout près des parois, la viscosité est faible. D'autre part, la figure 7 montre que la viscosité effective est d'autant plus élevée que le débit est grand.

\section{Approche analytique}

L'approche analytique a pour objectif d'établir une équation simple qui permet, à partir des données géométriques de la soupape, de calculer son débit pour un taux de détente et une levée donnés. 


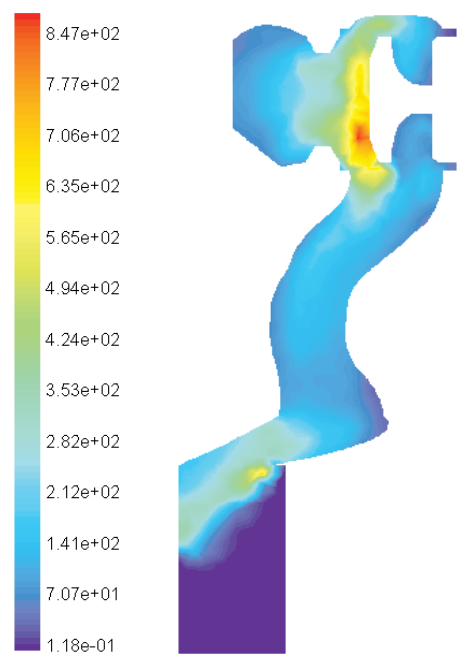

(a) Levée $8 \mathrm{~mm}$ - Débit $50 \mathrm{~g} / \mathrm{s}$

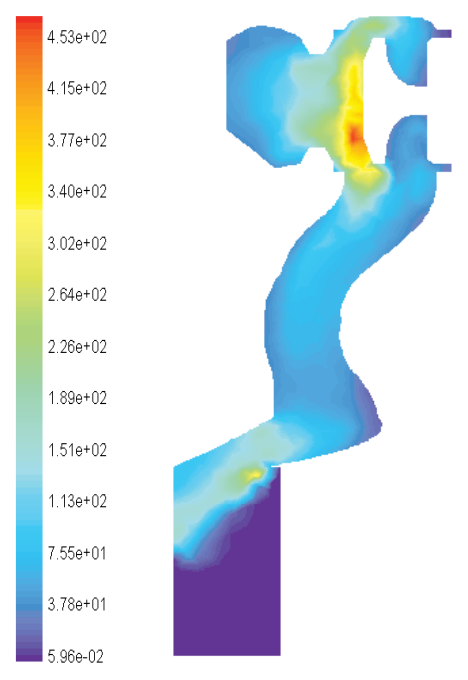

(c) Levée $8 \mathrm{~mm}$ - Débit $30 \mathrm{~g} / \mathrm{s}$
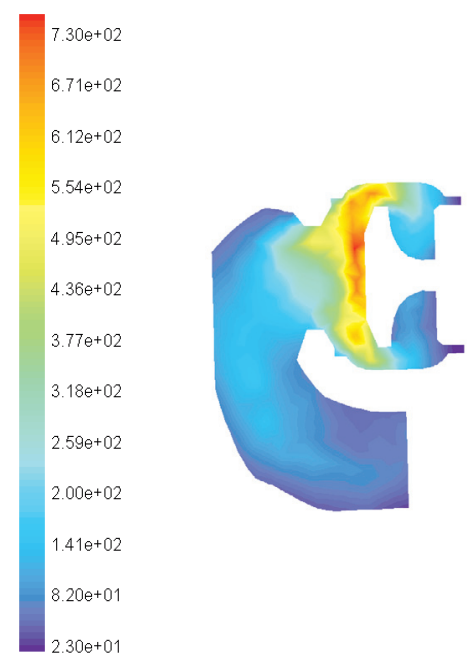

(b) Levée $8 \mathrm{~mm}$ - Débit $50 \mathrm{~g} / \mathrm{s}$

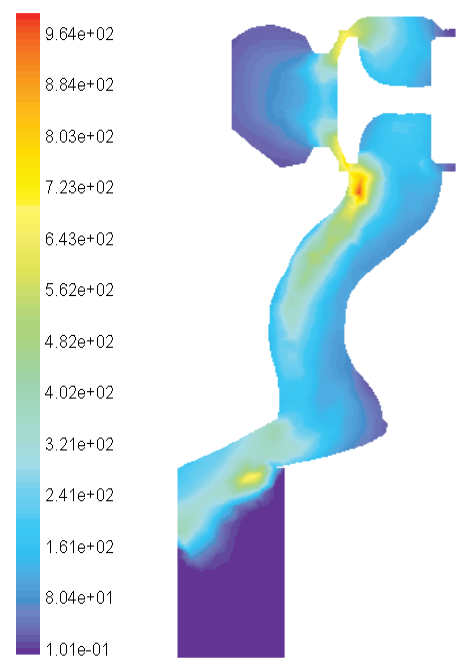

(d) Levée 1,6 mm - Débit $50 \mathrm{~g} / \mathrm{s}$

Fig. 5. Isocontours de l'énergie cinétique turbulente $\left(\mathrm{m}^{2} \cdot \mathrm{s}^{-2}\right)$.

Le débit massique de l'air à travers la soupape de décharge peut s'écrire :

$$
q_{\mathrm{m}}=\sigma S \sqrt{2 \bar{\rho}\left(p_{\text {ie }}-p_{\text {is }}\right)}
$$

D'autre part, la section $S$ de passage des gaz à travers la soupape est définie par :

$$
S=\pi D_{\mathrm{t}} H \cos \psi
$$

À partir de l'équation (11), la perte de pression d'arrêt à travers la soupape peut s'écrire :

$$
p_{\text {ie }}-p_{\text {is }}=\frac{q_{\mathrm{m}}^{2}}{2 \bar{\rho} \sigma^{2} S^{2}}
$$

Pour tenir compte de la compressibilité du gaz, la masse volumique moyenne $\bar{\rho}$ de l'équation (13) peut être définie par :

$$
\bar{\rho}=\frac{\rho_{\mathrm{ie}}+\rho_{\mathrm{is}}}{2}
$$

Par ailleurs, le premier principe de la thermodynamique stipule que l'enthalpie d'arrêt se conserve au cours de l'écoulement adiabatique et permanent à travers la soupape, d'où :

$$
T_{\mathrm{ie}}=T_{\mathrm{is}}
$$

À partir des équations (14) et (15), et en utilisant la loi des gaz parfaits, la masse volumique moyenne $\bar{\rho}$ s'écrit :

$$
\bar{\rho}=\frac{p_{\mathrm{ie}}+p_{\mathrm{is}}}{2 R T_{\mathrm{ie}}}
$$

D'autre part, en utilisant les équations (13) et (16), nous obtenons :

$$
p_{\mathrm{ie}}^{2}-p_{\mathrm{is}}^{2}=\frac{R T_{\mathrm{ie}} q_{\mathrm{m}}^{2}}{\sigma^{2} S^{2}}
$$



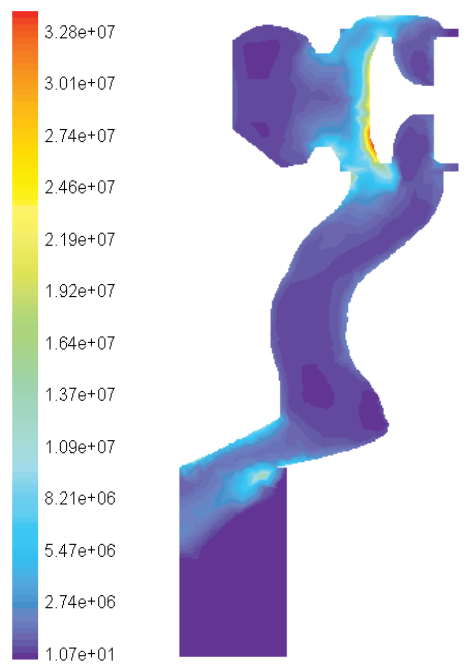

(a) Levée $8 \mathrm{~mm}$ - Débit $50 \mathrm{~g} / \mathrm{s}$

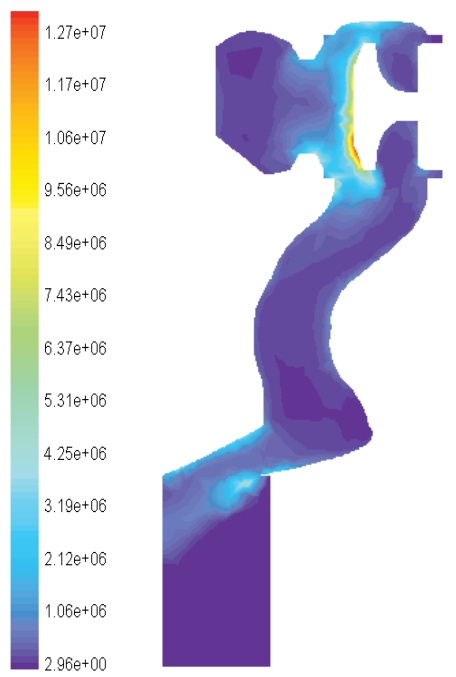

(c) Levée $8 \mathrm{~mm}$ - Débit $30 \mathrm{~g} / \mathrm{s}$
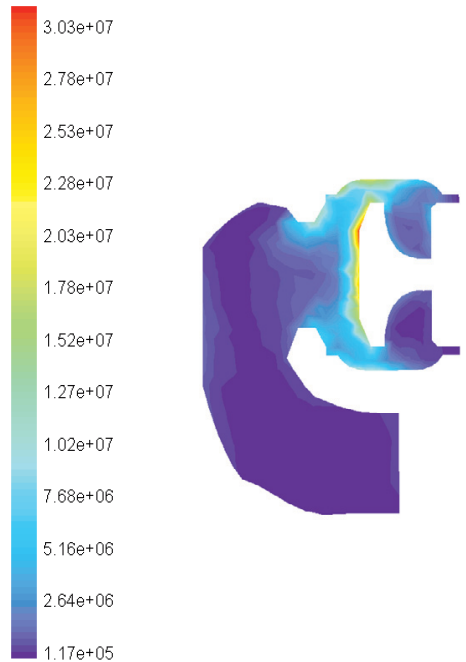

(b) Levée $8 \mathrm{~mm}$ - Débit $50 \mathrm{~g} / \mathrm{s}$
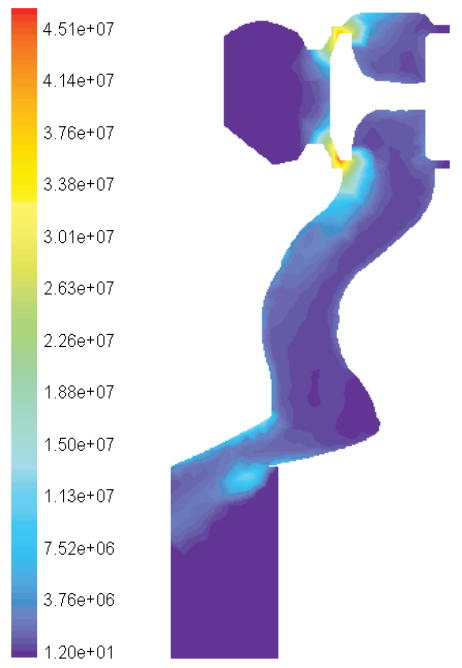

(d) Levée 1,6 mm - Débit $50 \mathrm{~g} / \mathrm{s}$

Fig. 6. Isocontours du taux de dissipation de l'énergie cinétique turbulente $\left(\mathrm{m}^{2} \cdot \mathrm{s}^{-3}\right)$.

Après avoir divisé l'équation (17) par $p_{\mathrm{ie}}^{2}$, et en introduisant la constante de la soupape $k_{\mathrm{s}}$, nous en déduisons le débit réduit $D_{\mathrm{r}}$ des gaz :

$$
D_{\mathrm{r}}=\frac{k_{\mathrm{s}}}{\sqrt{R}} H \sigma \sqrt{1-\frac{1}{\tau_{\mathrm{d}}^{2}}}
$$

avec $k_{\mathrm{s}}=\pi D_{\mathrm{t}} \cos \psi$. Cette constante ne dépend que des caractéristiques géométriques de la soupape.

Cette équation permet de calculer, à partir des données géométriques de la soupape et du coefficient de débit $\sigma$, la courbe caractéristique de fonctionnement donnant le débit réduit $D_{\mathrm{r}}$ des gaz, pour une levée $H$ et un taux de détente $\tau_{\mathrm{d}}$ donnés.

L'équation (18) repose donc sur la connaissance du coefficient de débit $\sigma$ de la soupape. À partir des résultats locaux de l'écoulement obtenus dans l'étude numérique développée à la section 2 , et en posant $\beta=\frac{S}{S_{\mathrm{t}}}$, la figure 8 montre l'évolution du coefficient de débit $\sigma$ en fonction du rapport $\beta$. D'après cette figure, le coefficient de débit $\sigma$ peut être corrélé par régression linéaire comme suit :

$$
\sigma=0,43-0,32 \operatorname{Ln} \beta
$$

\section{Validations expérimentales}

Pour la validation de notre travail de modélisations aussi bien numérique qu'analytique, nous avons utilisé un banc d'essai (Fig. 9), et nous l'avons équipé d'un turbocompresseur pour automobile de type KKK à soupape de décharge de type NW20.

Le rotor du turbocompresseur a été bloqué. De même, deux obturateurs ont été implantés. Le premier est placé 

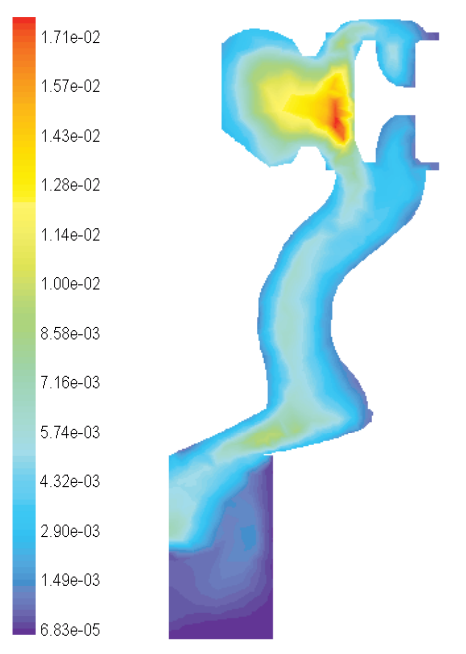

(a) Levée $8 \mathrm{~mm}$ - Débit $50 \mathrm{~g} / \mathrm{s}$

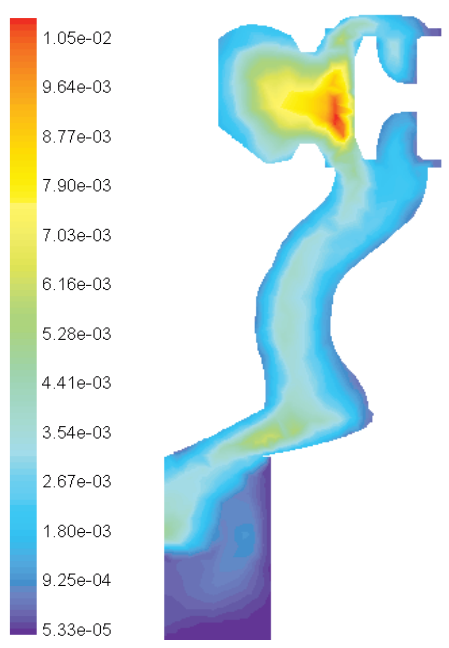

(c) Levée $8 \mathrm{~mm}$ - Débit $30 \mathrm{~g} / \mathrm{s}$
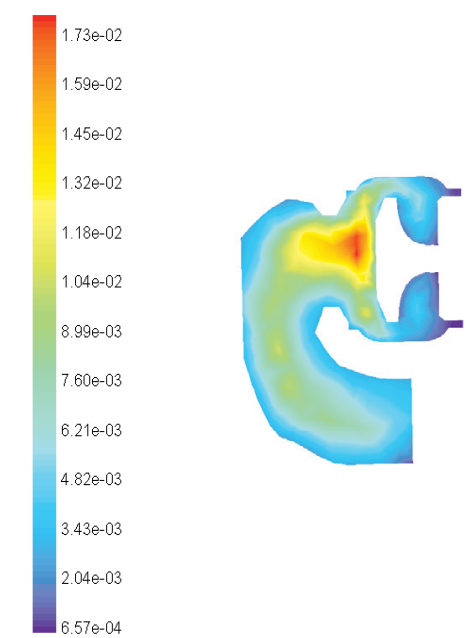

(b) Levée $8 \mathrm{~mm}$ - Débit $50 \mathrm{~g} / \mathrm{s}$
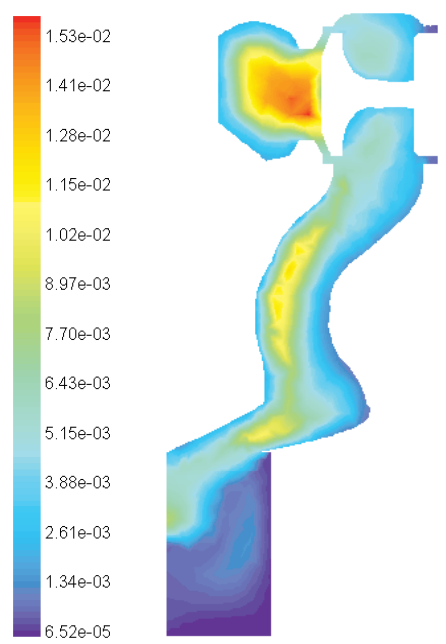

(d) Levée 1,6 mm - Débit $50 \mathrm{~g} / \mathrm{s}$

Fig. 7. Isocontours de la viscosité effective $\left(\mathrm{kg} \cdot \mathrm{m}^{-1} \cdot \mathrm{s}^{-1}\right)$.

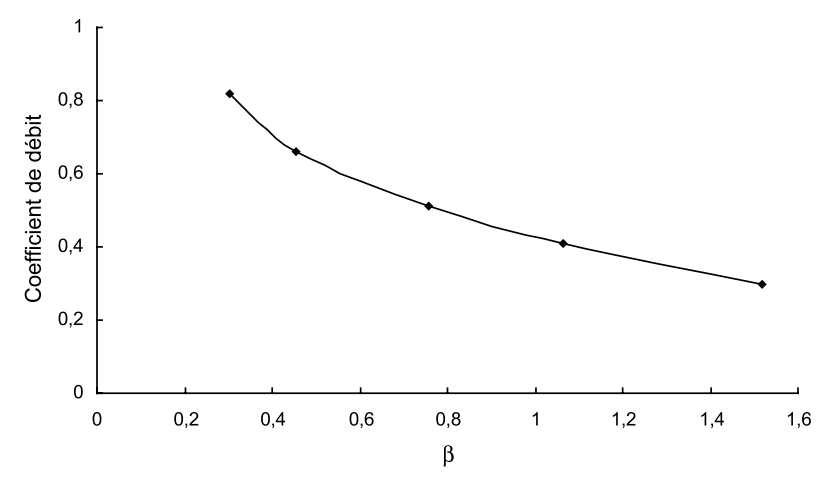

Fig. 8. Évolution du coefficient du débit de la soupape en fonction de $\beta$.

à l'entrée de la volute de la turbine; ainsi, tout l'air comprimé est dérivé directement vers la soupape. Le deuxième obturateur situé dans le diffuseur de la turbine, empêche le reflux d'air vers la turbine.

La mesure du débit s'effectue à l'aide d'un diaphragme et en appliquant la relation suivante :

$$
q_{\mathrm{m}}=C_{\mathrm{d}} s \sqrt{2 \rho \Delta p}
$$

$C_{\mathrm{d}}$ et $\Delta p$ étant respectivement le coefficient de débit et la pression différentielle du diaphragme. Pour calculer $C_{\mathrm{d}}$, nous avons étudié numériquement l'écoulement turbulent des fluides compressibles à travers le diaphragme. La valeur obtenue de $C_{\mathrm{d}}$ est de 0,608 .

Pour quatre levées de la soupape de décharge, la figure 10 compare les courbes caractéristiques de fonctionnement : débit réduit-taux de détente, que nous avons obtenu par voies numérique, analytique et expérimentale, avec celles obtenues par Bardez [15] par voie expérimentale. 


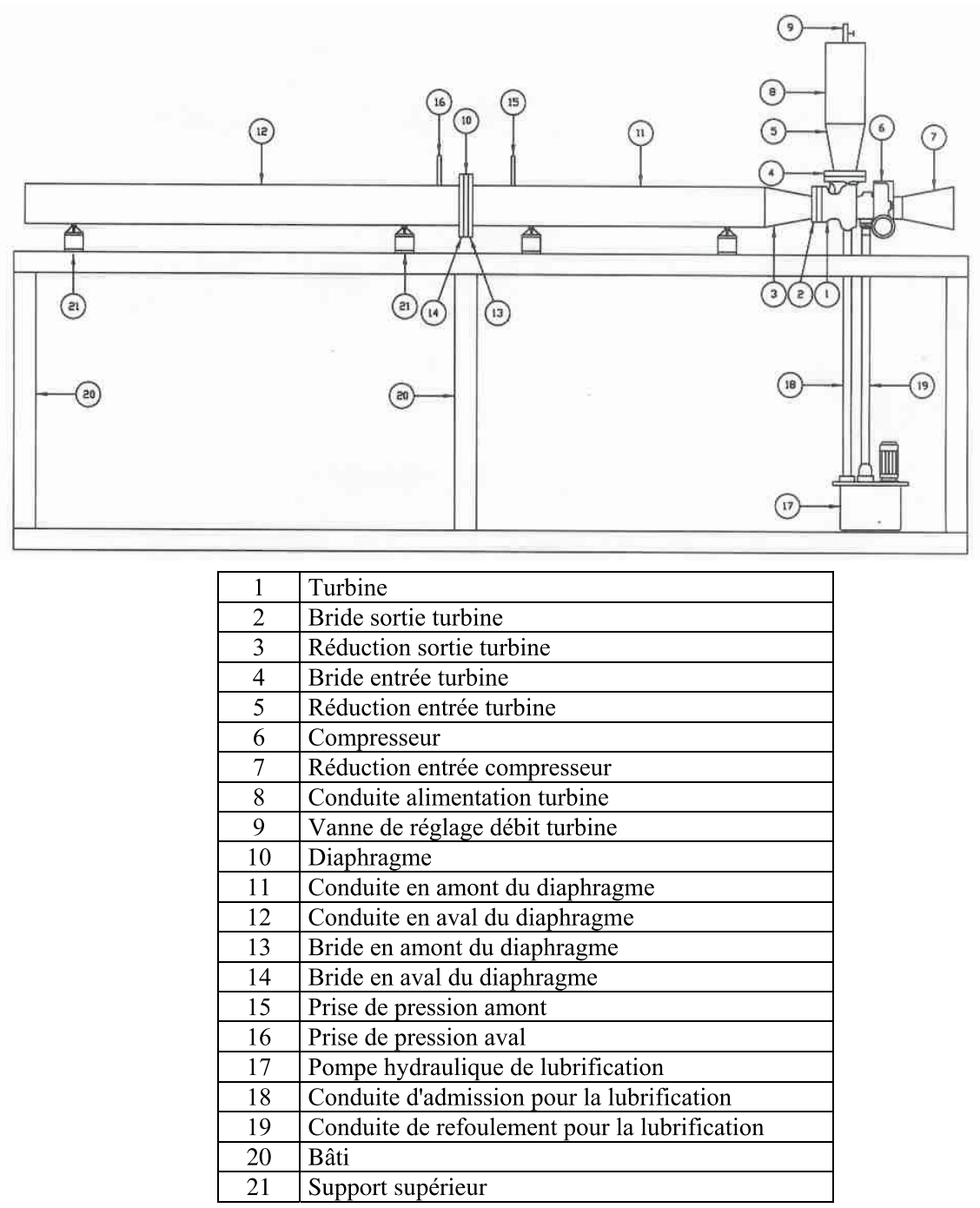

Fig. 9. Dessin descriptif du banc d'essai.

Cette figure montre que la concordance est très bonne pour les faibles levées et les faibles taux de détente, et que les résultats obtenus sont dans l'ensemble satisfaisants. En effet, par rapport à l'expérience, l'écart moyen $E_{\mathrm{m}}$ est raisonnable et est de l'ordre de $6 \%$.

\section{Conclusion}

Une étude numérique, analytique et expérimentale de l'écoulement turbulent des fluides compressibles à travers une soupape de décharge de turbocompresseur de suralimentation, est présentée.

La modélisation numérique a permis de fournir une connaissance fine des structures de l'écoulement, et de prédire à partir des résultats locaux de l'écoulement, les courbes caractéristiques de fonctionnement de la soupape ainsi que l'évolution du coefficient de débit en fonction de sa levée. Nous avons étudié en particulier l'influence du débit et de l'ouverture de la soupape sur les caractéristiques locales de l'écoulement ainsi que celles de la turbulence, et nous avons montré que ces caractéristiques dépendent fortement de la levée.

De même, une modélisation analytique a été établie à partir des données géométriques de la soupape. Elle se traduit par une équation simple. Grâce à cette équation, il est possible de construire un réseau donnant, pour une levée quelconque de la soupape, le débit à travers la soupape en fonction du taux de détente. D'autre part, pour une première estimation des performances d'un moteur turbosuralimenté aux forts régimes, cette modélisation permet au motoriste d'éviter les interpolations et les extrapolations dans le cas de l'utilisation des courbes caractéristiques expérimentales de la soupape, ainsi que les calculs longs et onéreux des approches locales multidimensionnelles.

Pour valider nos calculs, des expériences ont été menées sur un banc d'essai turbocompresseur. Ces essais sont limités à des taux de détente de l'ordre de 1,8.

La comparaison des courbes caractéristiques de fonctionnement obtenues à partir de notre travail avec celles des travaux expérimentaux antérieurs, a montré une 
Débit réduit $\left(\mathrm{kg} \cdot \mathrm{K}^{0.5} \cdot \mathrm{Pa}^{-1} \cdot \mathrm{s}^{-1}\right)$

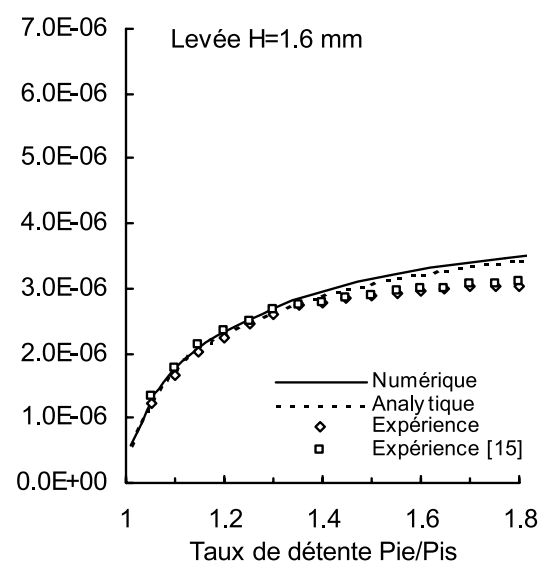

Débit réduit $\left(\mathrm{kg} \cdot \mathrm{K}^{0.5} \cdot \mathrm{Pa}^{-1} \cdot \mathrm{s}^{-1}\right)$

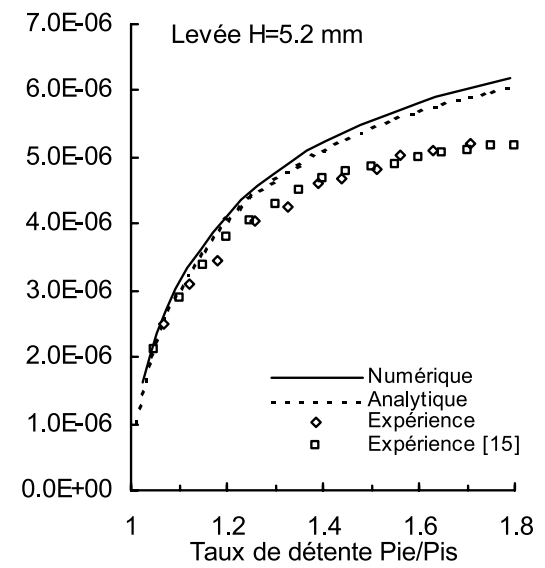

Débit réduit $\left(\mathrm{kg} \cdot \mathrm{K}^{0.5} \cdot \mathrm{Pa}^{-1} \cdot \mathrm{s}^{-1}\right)$
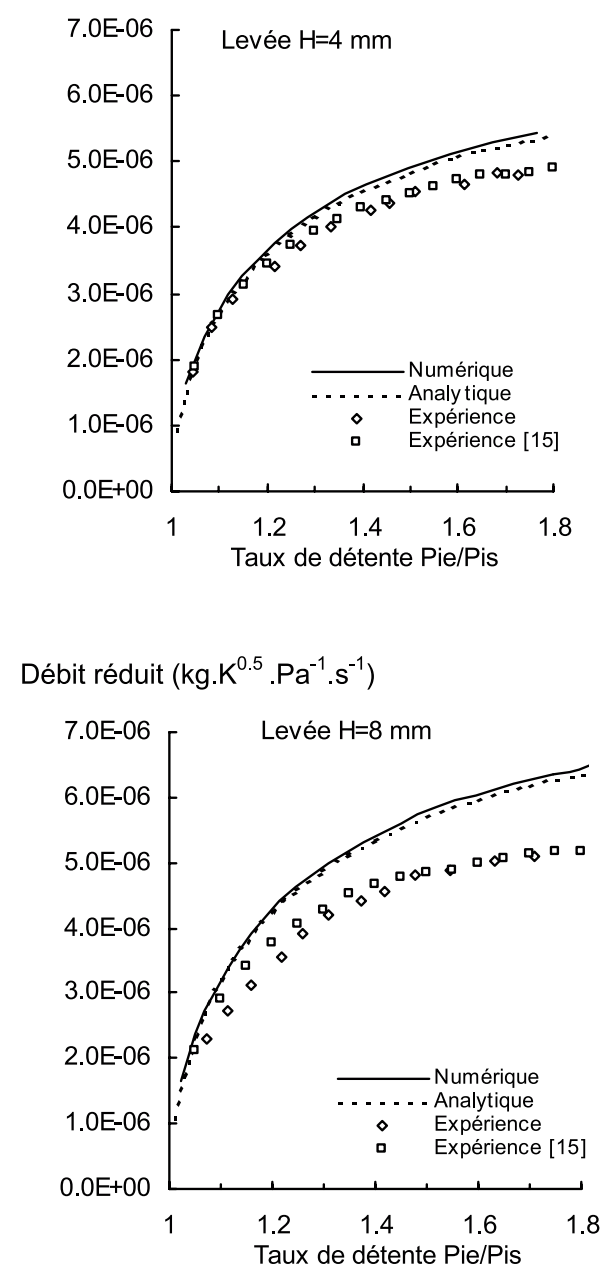

Fig. 10. Comparaison des courbes de fonctionnement de la soupape de décharge.

bonne concordance. Par ailleurs, aux grandes levées de la soupape, les débits réduits obtenus par les modèles sont légèrement plus élevés en comparaison avec les expériences, pour les points de fonctionnement à grands taux de détente.

Cependant, il sera utile d'étudier la zone des petites ouvertures de la soupape. De même, pour généraliser cette étude, il sera intéressant de faire une transposition à d'autres systèmes de dérivation des gaz d'échappement.

Remerciements. Nous tenons à remercier Messieurs Michel Toussaint et Pierre Podevin, Ingénieurs de Recherche au Laboratoire de Turbomachines au Conservatoire National des Arts et Métiers de Paris, pour leur précieuse collaboration.

\section{Références}

[1] R. Boussarhane, Caractérisation du champ de vitesses à la sortie d'une turbine de turbocompresseur, Thèse de $3^{\mathrm{e}}$ cycle, Université Paris 6, 1986
[2] N. Schorn, F. Pischinger, H. Schulte, Computer simulation of turbocharged Diesel engines under transient conditions, Copyright Society of Automotive Engineers, 1987, pp. $1-17$

[3] J. Jullien, H. Gayvallet, G. Papachristos, Modélisation d'une turbine centripète de suralimentation, Entropie 134 (1987) 19-26

[4] F. Thomas, Contribution à l'étude des performances d'une petite turbine de suralimentation en régime stationnaire, Thèse de Doctorat de l'ENSAM, Paris, 1987

[5] J. Friberg, M. Mahieddinne, M. Toussaint, M. Frelin, Prévision des caractéristiques des turbomachines. Application à une pompe centrifuge, un compresseur centrifuge et une turbine centripète, Revue Française de Mécanique 4 (1988) 55-66

[6] S. Fèvre, Application de la vélocimétrie Laser Doppler à des mesures de vitesses dans une petite turbine de suralimentation, Thèse de doctorat, Université Paris 6, 1990

[7] J. Lavy, Q.C. Duan, J. Jullien, G. Bois, Mesure des caractéristiques de fonctionnement de turbines de turbocompresseurs en régime stationnaire et pulsé, Revue Française de Mécanique 3 (1990) 217-223 
[8] M. Mseddi, Étude du couplage en régime quasistationnaire d'un turbocompresseur de suralimentation avec un moteur Diesel, Thèse de doctorat, Université Paris 6, 1990

[9] M. Frelin, Prévision des caractéristiques d'une turbine radiale à partir des données géométriques, Thèse de doctorat, Université de Paris 6, 1991

[10] M. Frelin, P. Podevin, Modélisation des petites turbines centripètes, Revue Française de Mécanique 3 (1992) 257 265

[11] A.A. Hammoud, Influence de l'écoulement pulsé sur le comportement d'une petite turbine de suralimentation, Thèse de doctorat de l'ENSAM, Paris, 1992

[12] P. Podevin, G. Descombes, P. Marez, F. Dubois, A study of turbocharged Diesel engine during sudden acceleration, sept-up and exploitation of a specific test rig, Fall technical conference of the ASME International Combustion Engine Division, October 1999, USA, pp. 1-7
[13] P. Podevin, G. Descombes, M. Pluviose, Simulation of a turbocharged Diesel engine, European Automotive Congress, Vehicule Systems Technology For the next Century, Barcelona, Spain, June-July 1999, pp. 1-10

[14] M. Mseddi, M. Baccar, H. Kchaou, M.S. Abid, Modélisation des turbines radiales de suralimentation, Mécanique \& Industries 3 (2002) 35-44

[15] L. Bardez, Étude de l'influence de la position de la soupape de décharge sur le fonctionnement d'un turbocompresseur de moteur d'automobile, Mémoire d'Ingénieur CNAM, 1990

[16] B.E. Launder, D.B. Spalding, Lectures in mathematical models of turbulence, Academic Press, London, England, 1972

[17] B.E. Launder, D.B. Spalding, The numerical computation of turbulents flows, Computer Methods in Applied Mechanics and Engineering 3 (1974) 269-289 